\title{
Comprehensive assessment of the association between topoisomerase single nucleotide polymorphism and doxorubicin cytotoxicity
}

\author{
Tana Takacova ${ }^{1}$, Jurgen Brockmoller ${ }^{2}$, Markus Schirmer ${ }^{3}$ \\ ${ }^{I}$ Department of Haematology, Oncology and Clinical Immunology, University of Dusseldorf, Germany, ${ }^{2}$ Institute of \\ Clinical Pharmacology, Georg-August University, Gottingen, Germany, ${ }^{3}$ Clinic for Radiotherapy and Radiooncology, \\ Georg-August University, Gottingen, Germany
}

\section{Background:}

The archetypical anthracycline doxorubicin (DOX) is highly effective and thus still widely used in oncology. However, inter-individual drug response is variable and a substantial contribution of inherited genetic variability is assumed.

Methods:

Lymphoblastoid cell lines (LCLs) were employed as model for rapidly proliferating cells. A total of 184 LCLs of unrelated Caucasians split at a $2: 1$ ratio into training $(n=120)$ and a test set $(n=64)$ were used. The LCLs were exposed to seven serial dilutions of DOX and a drug-free control. Upon incubation for $48 \mathrm{~h}$ cytotoxicity was assessed by fluorescenceactivated cell sorting. A four-parametric logistic regression model was applied to determine EC50 values for cytotoxicity of DOX. Comprehensive genetic variability of the employed LCLs was available from public databases. Association testing with the EC50 of DOX was performed for the five genes encoding topoisomerases in human (TOP1, TOP2A, TOPB, TOP3A, TOP3B) as these enzymes represent a major target of DOX. After filtering for a minor allele frequency of at least $5 \%$ in Caucasians and a linkage disequilibrium of $\mathrm{r} \& \# 178 ;<0.80$ a total number of 477 polymorphic sites in the denoted genes (including five kilobases of flanking regions on either side) remained for the analysis with the EC50 of DOX.

Results:

Reproducibility of the assay was proven in 27 LCLs measured at two independent time points with a high correlation of $\mathrm{r}$ $=0.80(\mathrm{p}=6 \mathrm{x} 10-7)$. In the training set, 20 out of the evaluated 477 genetic markers of the five human topoisomerase genes were associated with EC50 of DOX at $\mathrm{p}<0.05$. Upon adjustment for multiplicity testing association of one genetic polymorphism located in the 17th intron of the TOP3A gene was confirmed in the test set. Presence of the minor allele (frequency $9.5 \%$ in Caucasians) was coincident with higher sensitivity toward DOX reflected by $30 \%$ lower EC50 values both in the training and the test set.

Conclusion:

We observed a novel statistically significant association of a genetic polymorphism in the TOP3A gene with cytotoxicity of DOX, which may be valuable in future for individualized therapies or drug dose adjustments to improve therapeutic efficacy and safety when administering DOX. 